\title{
Safety and Considerations of the COVID-19 Vaccine Massive Deployment
}

\author{
Junwei $\mathrm{Li}^{1,2} \cdot$ Mingyue Song ${ }^{1,2} \cdot$ Deyin $\mathrm{GuO}^{3}(\mathrm{D}) \cdot$ Yongxiang $\mathrm{Yi}^{1,2}$ (D)
}

Received: 20 February 2021 / Accepted: 26 April 2021 / Published online: 1 June 2021

(c) Wuhan Institute of Virology, CAS 2021

Emerging and re-emerging infectious diseases represent a significant and growing cause of morbidity and mortality. Since vaccination campaigns were carried out, several infectious diseases have been controlled successfully, such as smallpox and poliomyelitis, making vaccination one of the most reliable and cost-effective public health interventions. In the long run, vaccine coverage, whether high or low, has been shown to yield important public health benefits (Weycker et al. 2005). Therefore, vaccines play an important role in increasing population immunity, preventing severe infectious diseases, and reducing the ongoing public health crisis. Due to herd immunity and consequent herd protection induced by vaccines, deployment of mass vaccination is vital to prevent and control the transmission of the emerging infectious diseases directly or indirectly.

In early 2020, the coronavirus disease 2019 (COVID-19) caused by severe acute respiratory syndrome coronavirus 2 (SARS-CoV-2), has spread rapidly worldwide, developing into a global COVID-19 disease pandemic. Hitherto, it has resulted in more than hundred millions of infections and three million deaths. Such a grave situation has made the development of a safe and effective COVID-19 vaccine imperative and urgent as a primary tool to prevent and eventually contain the spread of SARS-CoV-2. However, developing, scaling-up mass production, approval and

Yongxiang Yi

ian0126@126.com

$\triangle$ Deyin Guo

guodeyin@mail.sysu.edu.cn

1 Department of Infectious Diseases, The Second Hospital of Nanjing, The Affiliated Hospital of Nanjing University of Chinese Medicine, Nanjing 210003, China

2 Public Health and Therapy Center of Nanjing, Nanjing 211113, China

3 MOE Key Laboratory of Tropical Disease Control, Centre for Infection and Immunity Study, School of Medicine, Sun YatSen University, Shenzhen 518107, China distribution of COVID-19 vaccines rapidly in this global pandemic setting is challenging as it requires many activities, sequential process with pre-clinical testing, phased clinical trials, planned production, evaluation and followingup surveillance and faces much uncertainty. Being surprised, in this competition between spread and containment of SARS-CoV-2, groundbreaking universal effort in the development of vaccines against SARS-CoV-2 has shown great success unparalleled in the history of human vaccine development with an unprecedented pace thus far. Since the announcement of the genetic sequence of SARS-CoV-2 on January 12, 2020, it took only two months to the start of phase I clinical trial of candidate vaccines. On March 16, 2020, vaccine mRNA-1273, which encodes the spike protein (S protein) of SARS-CoV-2, developed by Moderna and the Vaccine Research Center (VRC) of the National Institute of Allergy and Infectious Diseases (NIAID), entered phase I clinical trial. On the same day, a non-replicating adenovirus type 5 vector of COVID-19 vaccine was announced to enter phase I clinical trial conducted by CanSino Biological Inc./ Beijing Institute of Biotechnology. Currently, more than one hundred of COVID-19 vaccines are under ongoing doubleblinded, randomized, and controlled clinical evaluation. Some of these have shown good safety and immunogenicity, and among them, more than 60 of these are being evaluated in phase III clinical efficacy studies globally.

Recently, integrated analysis of phase I, II, and III trials of COVID-19 vaccines was published one after another. These results showed that most COVID-19 vaccines provided protection among the vaccinated participants (Polack et al. 2020; Voysey et al. 2020). Based on the promising clinical data of COVID-19 vaccines, the United Kingdom (UK) has given approval first for use of COVID-19 vaccine, BNT162b2, by following a thorough review carried out by the Medicines and Healthcare products Regulatory Agency (MHRA) on December 1, 2020. Another mRNA vaccine, mRNA-1273, was authorized by the Food and Drug Administration (FDA) that issued an emergency use authorization (EUA) for general vaccination among health 
care workers in the United States (USA) on the same day. On December 11, 2020, the BNT162b2 mRNA vaccine was approved in the USA. With accumulated data on clinical phase III of COVID-19 vaccines presenting the efficacy and safety, such vaccines have been deployed in massive vaccination in more and more countries as emerging tools to constrain the spread of SARS-CoV-2, including Russia, Iran, Brazil, Pakistan, Mexico, et al.. Among these countries, UK and USA preferred to mRNA vaccine and adenoviral-vectored COVID-19 vaccine. mRNA vaccine, belonging to the nucleic acid vaccine, is a new-concept vaccine developed in recent years. There are several main safety and efficacy advantages of the use of mRNA-based antiviral vaccines. mRNA vaccines minimize the potential risk of infection and insertion-induced mutagenesis of the host genome due to natural degradation in the cellular physiological environment. mRNA structural modifications improve its stability and translation efficacy. The high potency of mRNA-based vaccines capable of generating potent antiviral neutralizing antibodies with low-dose immunizations can induce strong immune responses by activating both $\mathrm{CD} 8+$ and $\mathrm{CD} 4+\mathrm{T}$ cells. Prompt and large-scale production of mRNA vaccine with the GMP standard will be possible to meet the sufficient vaccine supply required to treat mass populations (Yi et al. 2020; Zhang et al. 2019). All these factors make the mRNA vaccine more suitable for a rapid response to the emerging COVID-19 pandemic than DNA vaccines. Adenoviral-vector has been employed in the development of several viral vaccines, such as the licensed Ebola vaccine. Inactivated vaccines are established technology with a long history. Both are assumed to show high safety on the COVID-19 vaccines (Voysey et al. 2020). At present, the adenoviral-vectored COVID-19 vaccine and inactivated COVID-19 vaccine have also been approved emergently to be used in several countries. Nevertheless, with the rapid progress of COVID-19 vaccine development, concerns on vaccine safety, potential adverse events, efficacy, reliability and distribution have been raised by public.

\section{Vaccine-Associated Disease Enhancement (VADE)}

Initially, the rapid development of COVID-19 vaccine increased many of the theoretical concerns on vaccineassociated disease enhancement (VADE) for the long-term of vaccine safety as in the use of dengue vaccine and inactivated respiratory syncytial viral vaccine (Castilow et al. 2007; Halstead 2017; Haynes et al. 2020; Russell et al. 2019; Su et al. 2021). The profile of VADE is complicated and categorized into antibody-mediated disease enhancement (ADE) and Th2 cell dependent disease exacerbation (Su et al. 2021). Phenomenon of VADE has been observed on several viral vaccines and hinders these vaccine developments dramatically. A report on a post hoc analysis of Dengue vaccine (DENV) clinical trial showed that sero-negative children aged 2-8 years vaccinated DENV had an almost fivefold greater relative incidence of serotype 2 Dengue virus infections comparing to nonvaccinated control subjects (Sridhar et al. 2018). Furthermore, research showed that antibodies of influenza virus and Ebola virus also induced ADE following challenge with corresponding virus in vivo (Takada et al. 2003; Winarski et al. 2019). Research by Chen and others confirmed that vaccination with SARS-CoV vaccines also led to pulmonary immune-pathology in mice and monkeys on challenge with SARS-CoV (Liu et al. 2019; Tseng et al. 2012). However, the mechanism and magnitude of VADE are not still fully understood. An incident caused by a formalin-inactivated respiratory syncytial virus (FI-RSV) vaccine indicated that lack of viral neutralization and altered $\mathrm{T}$ cell responses contributed to VADE in the immunized individuals (Knipe et al. 2020). Recently, research showed that VADE is associated with antibody FcR-mediated internalization of infected viruses, complement activation and inflammatory cytokine release $(\mathrm{Su}$ et al. 2021). Fortunately, there is no evidence that SARSCoV-2 infects FcR-bearing cell types which can be infected by some other viruses with severe ADE, such as dengue virus and RSV (Su et al. 2021). The integrated clinical trial results of BNT162b2 showed that most participants had T helper type 1 (Th1) immune responses with balanced RBDspecific CD8+ and CD4+ T-cell, and absence of potentially deleterious $\mathrm{Th} 2$ immune response-related ADE. What is more, the detection of associated cytokines indicates a favorable Th1 profile that benefits the potent immune response (Sahin et al. 2020). It is encouraging that more and more research results showed that COVID-19 vaccines did not induce VADE in mice, hamster, or non-human primates, and it is most important, so far, during the expanding use of COVID-19 vaccine, there is no ADE reported with the expanding use of COVID-19 vaccines in human (Li et al. 2021). However, it is too early to reject the hypothesis that vaccination with COVID-19 vaccines barely cause ADE. Researches indicated ADE could happen at sub-neutralizing concentrations of the antibodies when cells were infected by HIV-1, DENV or Enterovirus 71 (EV71) (Chen et al. 2013; Durham et al. 2019; Meyer et al. 2011). With the weaning on immune response induced by current vaccination program of COVID-19 vaccines, there is stubborn potential of ADE caused when immunized people is infected by SARS-CoV-2. It is necessary to conduct long-term surveillance to collect the safety information on the vaccination of COVID-19 vaccines. 


\section{Side Effects}

During the clinical test of mRNA-1273 vaccines, side effects, including fatigue, chills, adverse events were dosedependent and were much common after the second immunization (Anderson et al. 2020). The safety profile of BNT162b2 was characterized by short-term, mild-tomoderate pain at the injection site, injection-site redness or swelling. Systemic symptoms such as fever, fatigue, headache, and muscle and joint pain have also been more common, and most have occurred during the first 24-48 h after vaccination (Polack et al. 2020). Serious adverse events including severe pain were observed occasionally. Most local reactions were disappeared with 1-2 days (Polack et al. 2020).

The data collected from mass vaccination with mRNA1273 and BNT162b2 indicate the anaphylactic rate is around 1/100000, higher than that of common vaccines (Castells and Phillips 2021). Anaphylaxis is a treatable condition with nonpermanent effects. Nevertheless, these reactions have raised fear about the risks of a new vaccine. Such safety signals highlight the need for a robust and proactive interpretation to define causal mechanisms, to identify populations at risk for such reactions, and to facilitate implementing strategies. Acute allergic reactions after vaccination might be caused by the vaccine antigen, residual nonhuman protein, or preservatives and stabilizers in the vaccine formulation, such as antibiotics, gelatin, and thimerosal in some licensed vaccines (Stone et al. 2019). There is therefore no prior experience that informs the likelihood or explains the mechanism of allergic reactions associated with mRNA vaccines. It is possible that some populations are at higher risk for non-IgE-mediated mastcell activation or complement activation related to either the lipid or the PEG-lipid component of the vaccine, substituted or modified nucleic acid, such as pseudouridine, or 5-methylcytidine (5 mC) (Pardi and Weissman 2017). Careful animal preclinical studies as well as intense monitoring of human clinical trials will be of critical importance to developing safe and effective anti-COVID-19 antibodies and vaccines.

\section{Vulnerable Populations}

In the fast-track approval of new vaccine against the emerging infectious disease, vaccine efficacy in underrepresented and vulnerable populations also remains an important issue. As the COVID-19 pandemic continues to unfold, there has been a widespread impact on health, in particular, including substantial mortality among older adults and those with pre-existing health conditions $(\mathrm{Li}$ et al. 2020; Wang et al. 2020). The evaluation of immune response stimulated by these COVID-19 vaccines must have special considerations for fragile people who are at increased risk and suffer severely due to COVID-19 (Pawelec and Weng 2020). SARS-CoV-2 infection affects all age groups, however, those over 60 years old are affected more severely. As the aging population is increasing globally, especially in developed countries, studies should be highlighted the age-related decrease in vaccine efficacy, which is due to a decline in the adaptive and innate immune response (Perfilyeva et al. 2020). Given the disproportionate mortality rate in people over the age of 60 , the elderly need to be considered carefully in vaccine trials to ensure safety. Vaccine efforts must take into consideration age-related issues to ensure effective control of infectious diseases during the mass deployment of COVID-19.

So far, a majority of vaccine trials have focused on healthy people between the ages of 18-65 years, excluding the elderly, pregnant women and children. Moreover, preexisting co-morbidities result in higher COVID-19-associated mortality in the geriatric population. Efforts are necessary to ensure a healthier life for the elderly as life expectancy is increasing (Koff and Williams 2020). Hence, while considering a vaccine candidate for older people, safety issues must be adequately addressed. If a vaccine does not become available soon, elderly and immune-compromised individuals with other comorbidities such as diabetes, hypertension, cancer, asthma, and cardiovascular abnormalities are more severely affected with a higher rate of case mortality.

\section{Vaccine Mistrust and Hesitancy}

Vaccine hesitancy was defined as a delay in acceptance or refusal of vaccination despite the availability of vaccination services, which was referred as one of the top ten threats to global health in 2019 by the World Health Organization (WHO). There is growing evidence of vaccine delays or refusals due to a lack of trust in the importance, safety, or effectiveness of vaccines, alongside persisting access issues. In recent years, concern about vaccine hesitancy is growing worldwide (Larson et al. 2014). In many countries, vaccine hesitancy and misinformation present substantial obstacles to achieving coverage and community immunity (Lane et al. 2018). Influenza vaccine program is a well-educated health program and influenza vaccine has been made available free of charge, however, only a limited proportion of the population accepted to be vaccinated against seasonal influenza. So far, people might need two doses of COVID-19 vaccines to build potent immunity and prevent infection by SARS$\mathrm{CoV}-2$ and the exact duration of the immunity induced by 
vaccination with COVID-19 vaccine is still unknown. Even suggestion of annual administration is made. People will feel upset if vaccination with COVID-19 vaccine is performed every year. Nobody knows what is the new problem brought by repetitive administration of COVID-19 vaccines. Additionally, variants of SARS-CoV-2 are emerging around the world and the impotence of current COVID-19 vaccine against current SARS-CoV-2 is assumed. This situation deteriorates the vaccine mistrust and hesitancy on COVID-19 vaccines furthermore. A report in Italy showed that $40.8 \%$ of the population reported the intention to be vaccinated against influenza next winter, and in this survey, there were $53.7 \%$ would accept to receive a potential COVID-19 vaccine among the involved 1055 Italians (La Vecchia et al. 2020). These data confirm a certain degree of vaccine mistrust, limited confidence, and a diffuse vaccine mistrust and hesitancy in Italy as well as in other countries, especially among less qualified workers.

A recent surveillance in Japan showed that only $65.7 \%$ of the participants indicated a willingness to be vaccinated, among them were older age groups, those in rural areas, and those with underlying medical conditions (Yoda and Katsuyama 2021). According to a recent global survey, the vaccine acceptance rate varies in different countries, with a result of $88.6 \%$ COVID-19 vaccine acceptance in China and $55 \%$ in Russia, indicating higher levels of trust in information from government and public health agents is crucial for universal COVID-19 vaccine coverage (Lazarus et al. 2020). A surveillance in France, Belgium and Canada on healthcare personnel who are at high risk of infection by SARS-CoV-2 to assess willingness attitudes, beliefs to get vaccinated with COVID-19 vaccine showed $71.6 \%$ acceptance of COVID-19 vaccine (Verger et al. 2021). Another surveillance on healthcare personnel showed only overall $57.5 \%$ of individuals expressed intent to receive COVID-19 vaccine in New York (Shaw et al. 2021). A surveillance on the medical students showed that only 53\% indicated they would participate in a COVID-19 vaccine trial and 23\% were unwilling to take a COVID-19 vaccine immediately upon FDA approval, highlighting the need for an educational curriculum about the safety and effectiveness to promote uptake of the COVID-19 vaccine (Lucia et al. 2020). Obviously, vaccine hesitancy can slow down the containment of COVID-19 pandemic. However, a recent report on a survey in 15 countries showed that an increasing portion of people are willing to be vaccinated with COVID-19 vaccine (Mega 2021). It is encouraging that the vaccine confidence is growing in some countries.

According to the public data, each shot of COVID-19 vaccine costs 15-25 USA dollars. In the developed countries, most people have the finance to afford the vaccination of COVID-19 vaccine, however, in the developing countries, most people in the poor life condition do not have the ability to pay the vaccination with COVID-19. This causes a question on the global vaccine equity and further worsens the acceptance of COVID-19 vaccines. Fortunately, several governments have announced vaccination with COVID-19 vaccine is free, such as in China, Japan, Russia, Saudi Arab, etc. This strategy may increase the vaccine trust and release the financial burden of averages.

\section{Strategies to Improve COVID-19 Vaccine Massive Immunization}

Given that disease diminishes as a result of massive vaccination, sometimes, public attention shifts from disease prevention to vaccine safety, in particular on such newly introduced vaccines. Public trust in newly introduced vaccines can be strengthened by monitoring vaccine safety. Accordingly, COVID-19 vaccine should be safe for all people, irrespective of age, gender, race, poverty and those with or without co-morbidities. The adverse reaction rate of a COVID-19 vaccine should be kept extremely low when it is distributed globally and surveillance of post-vaccination should be enforced. Safety surveillances should be conducted at all stages of product development, from selection and formulation of candidate vaccines through post-licensure studies and surveillance of adverse-event reports. Surveillance of adverse events following immunization will enable us to monitor the safety of immunization programs and thereby contribute to validating the immunization program. In this way, the undesirable adverse events and any inappropriate reports on adverse effects of the immunization program can be effectively managed and prevented. In addition, long-term research on the immune response stimulated by COVID-19 vaccines should be carried out to obtain comprehensive data for safe and effective COVID-19 vaccines. Hence, the expanding amount of knowledge is helpful to gain public trust and expand the vaccine coverage. As well, political efforts based on these surveillance programs can help make the country's vaccination program successful.

Before the COVID-19 vaccines with a temporary license have been used in China, the public health office has made several guidelines to enforce the safety of vaccine in the administration, including 30-min observation in the administration site and a report system to collect the adverse effects. In the United States, the government has set a Vaccine Adverse Event Reporting System (VAERS) to collect reports for post-vaccination adverse events or symptoms that occur after the administration of licensed vaccines many years ago. This system helps to improve public health dramatically. An analysis of the relationship 
between vaccines and reported adverse events between 1990-2013 indicates that adverse events or symptoms with high occurrence frequencies are positively correlated, and those most frequently occurred adverse symptoms are in many cases positively correlated with viral vaccines, especially flu vaccines, and no particular patterns are shown with live vs. inactive vaccines (Ren et al. 2019). These data will benefit the subsequent massive vaccination of COVID-19 vaccines, whether live or inactivated, even mRNA vaccine. The Vaccine Confidence Project (VCP) was launched by the London School of Hygiene and \&Tropical Medicine, aiming to develop systematic approaches to monitoring public confidence in vaccines and to inform policymakers and stakeholders of the changing trends and determinants of vaccine confidence across the globe.

Maintaining public confidence with a clear safety profile of vaccines to minimize vaccine hesitancy will be crucial. In practice, physicians should provide vaccine information statements to parents who are considering vaccine refusal or delay and direct them to credible sources of information. In the next period, millions of people globally will be exposed to new vaccines over the next several months, we must be prepared to develop strategies to maximize effectiveness and safety at an individual and a population level. Systemic post-vaccination surveillance and documentation are needed to conclude a strategic and systematic approach to vaccine safety. More data are needed across demographics. Post-crossover estimates of vaccine efficacy can provide insights about durability, identify waning efficacy, and identify late enhancement of disease.

Prevention of COVID-19 in other populations, such as younger adolescents, children, and pregnant women were not reported so far. Sometimes, syncope may occurespecially in adolescents. Vaccinees, in particular for adolescents, should be observed for 15 min after receiving these vaccines (Spencer et al. 2017). Due to the lack of data on clinical tests in young people, COVID-19 vaccines have not been recommended to people under 18 -year old. It is good news that Moderna has designed clinical trials to test mRNA-1273 in adolescents.

As well, people will be watching closely for as-yet unobserved signs of dangers. Fortunately, many countries set up programs to collect the serious symptoms after people receive the vaccine such as China, the United States, and the United Kingdom.

Currently, in China, after the extremely prudent evaluation, several kinds of people are not recommended to receive COVID-19 vaccine administration, including any people of the age under 18, people with any allergic history to components in vaccines, being fever, in acute and chronic illness, pregnant and lactating women, in genetic nervous-system disorder, with Guillain-Barre Syndrome,
HIV carrier, with autoimmune diseases, chronic cardiovascular comorbidities, known or suspected to have severe respiratory diseases, severe cardiovascular diseases, liver and kidney diseases, malignant tumors, people who use immuno-modulators such as anti-tumor drugs, SARS-CoV-2 infected, etc.

A safe and efficacious vaccine against SARS-CoV-2 will continue to contribute to the control of the COVID-19. Currently, most COVID-19 vaccines require prime and booster administration except for Adenovirus-5 COVID-19 Vaccines from CanSino Bio and Ad26.COV2.S vaccine provided by Johnson \& Johnson's Janssen. The speculated incompatibility of different types of COVID-19 vaccines also delays the acceptance of COVID-19 vaccines. It is better to test the possibility for combination of different types COVID-19 vaccines. With the approval of COVID-19 mRNA vaccines, mRNA-1273 and BNT162b2, to be used emergently against SARS-CoV-2, a lot of mRNA-based vaccines and drugs will bring up. Whether the exogenous mRNA is tolerable with repetitive administration should be investigated. With the extensive acceleration on expanding COVID-19 vaccine vaccination, the positive impact on the decline of SARS-CoV-2 transmission has been observed in Israel and United Arab Emirates (Mallapaty 2021). Even such success has been seen, continued follow-up of trial participants to best assess vaccine durability and potential delayed enhancement of disease still need to be enhanced. Although the use of COVID-19 vaccine may contain the transmission of SARA-CoV-2, control measures such as the use of masks, physical distancing, personal hygiene, testing of exposed or symptomatic persons, contact tracing, and isolation are still of pivotal importance in protecting personal and public health against COVID-19.

Acknowledgements This study was supported by Key Research and Development Program of Department of Health of Jiangsu (ZDB2020036).

\section{Compliance with Ethical Standards}

Conflict of interest The authors declare that they have no conflict of interest.

Animal and Human Rights Statement This article does not contain any studies with human or animal subjects performed by any of the authors.

\section{References}

Anderson EJ, Rouphael NG, Widge AT, Jackson LA, Roberts PC, Makhene M, Chappell JD, Denison MR, Stevens LJ, Pruijssers AJ, McDermott AB, Flach B, Lin BC, Doria-Rose NA, O'Dell S, Schmidt SD, Corbett KS, Swanson PA 2nd, Padilla M, Neuzil KM, Bennett H, Leav B, Makowski M, Albert J, Cross K, Edara VV, Floyd K, Suthar MS, Martinez DR, Baric R, Buchanan W, 
Luke CJ, Phadke VK, Rostad CA, Ledgerwood JE, Graham BS, Beigel JH; mRNA-1273 Study Group (2020) Safety and immunogenicity of SARS-CoV-2 mRNA-1273 vaccine in older adults. N Engl J Med 383:2427-2438

Castells MC, Phillips EJ (2021) Maintaining safety with SARS-CoV-2 vaccines. N Engl J Med 384:e37

Castilow EM, Olson MR, Varga SM (2007) Understanding respiratory syncytial virus (RSV) vaccine-enhanced disease. Immunol Res 39:225-239

Chen IC, Wang SM, Yu CK, Liu CC (2013) Subneutralizing antibodies to enterovirus 71 induce antibody-dependent enhancement of infection in newborn mice. Med Microbiol Immunol 202:259-265

Durham ND, Agrawal A, Waltari E, Croote D, Zanini F, Fouch M, Davidson E, Smith O, Carabajal E, Pak JE, Doranz BJ, Robinson M, Sanz AM, Albornoz LL, Rosso F, Einav S, Quake SR, McCutcheon KM, Goo L (2019) Broadly neutralizing human antibodies against dengue virus identified by single B cell transcriptomics. Elife 8:e52384

Halstead SB (2017) Dengvaxia sensitizes seronegatives to vaccine enhanced disease regardless of age. Vaccine 35:6355-6358

Haynes BF, Corey L, Fernandes P, Gilbert PB, Hotez PJ, Rao S, Santos MR, Schuitemaker H, Watson M, Arvin A et al (2020) Prospects for a safe COVID-19 vaccine. Sci Transl Med 12:eabe 0948

Knipe DM, Levy O, Fitzgerald KA, Muhlberger E (2020) Ensuring vaccine safety. Science 370:1274-1275

Koff WC, Williams MA (2020) Covid-19 and immunity in aging populations - a new research agenda. N Engl J Med 383:804-805

La Vecchia C, Negri E, Alicandro G, Scarpino V (2020) Attitudes towards influenza vaccine and a potential COVID-19 vaccine in Italy and differences across occupational groups, September 2020. Med Lav 111:445-448

Lane S, MacDonald NE, Marti M, Dumolard L (2018) Vaccine hesitancy around the globe: analysis of three years of WHO/ UNICEF joint reporting form data-2015-2017. Vaccine 36:3861-3867

Larson HJ, Jarrett C, Eckersberger E, Smith DM, Paterson P (2014) Understanding vaccine hesitancy around vaccines and vaccination from a global perspective: a systematic review of published literature, 2007-2012. Vaccine 32:2150-2159

Lazarus JV, Ratzan SC, Palayew A, Gostin LO, Larson HJ, Rabin K, Kimball S, El-Mohandes A (2020) A global survey of potential acceptance of a COVID-19 vaccine. Nat Med 27:225-228

Li P, Chen L, Liu Z, Pan J, Zhou D, Wang H, Gong H, Fu Z, Song Q, Min Q, Ruan S, Xu T, Cheng F, Li X (2020) Clinical features and short-term outcomes of elderly patients with COVID-19. Int J Infect Dis 97:245-250

Li D, Edwards RJ, Manne K, Martinez DR, Schäfer A, Alam SM, Wiehe K, Lu X, Parks R, Sutherland LL, Oguin TH, McDanal C, Perez LG, Mansouri K, Gobeil SMC, Janowska K, Stalls V, Kopp M, Cai F, Lee E, Foulger A, Hernandez GE, Sanzone A, Tilahun K, Jiang C, Tse LV, Bock KW, Minai M, Nagata BM, Cronin K, Gee-Lai V, Deyton M, Barr M, Von Holle T, Macintyre AN, Stover E, Feldman J, Hauser BM, Caradonna TM, Scobey TD, Moody MA, Cain DW, DeMarco CT, Denny TN, Woods CW, Petzold EW, Schmidt AG, Teng IT, Zhou T, Kwong PD, Mascola JR, Graham BS, Moore IN, Seder R, Andersen H, Lewis MG, Montefiori DC, Sempowski GD, Baric RS, Acharya P, Haynes BF, Saunders KO (2021) The functions of SARS-CoV-2 neutralizing and infection-enhancing antibodies in vitro and in mice and nonhuman primates. bioRxiv. https:// doi.org/10.1101/2020.12.31.424729

Liu L, Wei Q, Lin Q, Fang J, Wang H, Kwok H, Tang H, Nishiura K, Peng J, Tan Z, Wu T, Cheung KW, Chan KH, Alvarez X, Qin C, Lackner A, Perlman S, Yuen KY, Chen Z (2019) Anti-spike IgG causes severe acute lung injury by skewing macrophage responses during acute SARS-CoV infection. JCI Insight 4:e123158

Lucia VC, Kelekar A, Afonso NM (2020) COVID-19 vaccine hesitancy among medical students. J Public Health (oxf). https:// doi.org/10.1093/pubmed/fdaa230

Mallapaty S (2021) Are COVID vaccination programmes working? Scientists seek first clues. Nature. https://doi.org/10.1038/ d41586-021-00140-w

Mega ER (2021) Trust in COVID vaccines is growing. https://www. nature.com/articles/d41586-021-00368-6. Accessed 10 Feb 2021

Meyer K, Banerjee A, Frey SE, Belshe RB, Ray R (2011) A weak neutralizing antibody response to hepatitis $\mathrm{C}$ virus envelope glycoprotein enhances virus infection. PLoS ONE 6:e23699

Pardi N, Weissman D (2017) Nucleoside modified mRNA vaccines for infectious diseases. Methods Mol Biol 1499:109-121

Pawelec G, Weng NP (2020) Can an effective SARS-CoV-2 vaccine be developed for the older population? Immun Ageing 17:8

Perfilyeva YV, Karalnik BV, Ostapchuk YO, Kali A, Tleulieva RT, Abdolla N, Krasnoshtanov VK, Belyaev NN (2020) Age-related decline in vaccination efficacy: the potential role of myeloid derived suppressor cells. Adv Gerontol 33:785-795 (In Russian)

Polack FP, Thomas SJ, Kitchin N, Absalon J, Gurtman A, Lockhart S, Perez JL, Pérez Marc G, Moreira ED, Zerbini C, Bailey R, Swanson KA, Roychoudhury S, Koury K, Li P, Kalina WV, Cooper D, Frenck RW Jr, Hammitt LL, Türeci Ö, Nell H, Schaefer A, Ünal S, Tresnan DB, Mather S, Dormitzer PR, Şahin U, Jansen KU, Gruber WC; C4591001 Clinical Trial Group (2020) Safety and efficacy of the BNT162b2 mRNA Covid-19 vaccine. N Engl J Med 383:2603-2615

Ren JJ, Sun T, He Y, Zhang Y (2019) A statistical analysis of vaccine-adverse event data. BMC Med Inform Decis Mak 19:101

Russell MS, Creskey M, Muralidharan A, Li C, Gao J, Chen W, Larocque L, Lavoie JR, Farnsworth A, Rosu-Myles M, Hashem AM, Yauk CL, Cao J, Van Domselaar G, Cyr T, Li X (2019) Unveiling integrated functional pathways leading to enhanced respiratory disease associated with inactivated respiratory syncytial viral vaccine. Front Immunol 10:597

Sahin U, Muik A, Derhovanessian E, Vogler I, Kranz LM, Vormehr M, Baum A, Pascal K, Quandt J, Maurus D, Brachtendorf S, Lörks V, Sikorski J, Hilker R, Becker D, Eller AK, Grützner J, Boesler C, Rosenbaum C, Kühnle MC, Luxemburger U, Kemmer-Brück A, Langer D, Bexon M, Bolte S, Karikó K, Palanche T, Fischer B, Schultz A, Shi PY, Fontes-Garfias C, Perez JL, Swanson KA, Loschko J, Scully IL, Cutler M, Kalina W, Kyratsous CA, Cooper D, Dormitzer PR, Jansen KU, Türeci Ö (2020) COVID-19 vaccine BNT162b1 elicits human antibody and TH1 T cell responses. Nature 586:594-599

Shaw J, Stewart T, Anderson KB, Hanley S, Thomas SJ, Salmon DA, Morley C (2021) Assessment of U.S. health care personnel (HCP) attitudes towards COVID-19 vaccination in a large university health care system. Clin Infect Dis. https://doi.org/10. 1093/cid/ciab054

Spencer JP, Trondsen Pawlowski RH, Thomas S (2017) Vaccine adverse events: separating myth from reality. Am Fam Physician 95:786-794

Sridhar S, Luedtke A, Langevin E, Zhu M, Bonaparte M, Machabert T, Savarino S, Zambrano B, Moureau A, Khromava A, Moodie Z, Westling T, Mascareñas C, Frago C, Cortés M, Chansinghakul D, Noriega F, Bouckenooghe A, Chen J, Ng SP, Gilbert PB, Gurunathan S, DiazGranados CA (2018) Effect of dengue serostatus on dengue vaccine safety and efficacy. N Engl J Med 379:327-340 
Stone CA Jr, Rukasin CRF, Beachkofsky TM, Phillips EJ (2019) Immune-mediated adverse reactions to vaccines. $\mathrm{Br} \mathrm{J}$ Clin Pharmacol 85:2694-2706

Su S, Du L, Jiang S (2021) Learning from the past: development of safe and effective COVID-19 vaccines. Nat Rev Microbiol 19:211-219

Takada A, Feldmann H, Ksiazek TG, Kawaoka Y (2003) Antibodydependent enhancement of Ebola virus infection. J Virol 77:7539-7544

Tseng CT, Sbrana E, Iwata-Yoshikawa N, Newman PC, Garron T, Atmar RL, Peters CJ, Couch RB (2012) Immunization with SARS coronavirus vaccines leads to pulmonary immunopathology on challenge with the SARS virus. PLoS ONE 7:e35421

Verger P, Scronias D, Dauby N, Adedzi KA, Gobert C, Bergeat M, Gagneur A, Dubé E (2021) Attitudes of healthcare workers towards COVID-19 vaccination: a survey in France and Frenchspeaking parts of Belgium and Canada, 2020. Euro Surveill 26:2002047

Voysey M, Clemens SAC, Madhi SA, Weckx LY, Folegatti PM, Aley PK, Angus B, Baillie VL, Barnabas SL, Bhorat QE, Bibi S, Briner C, Cicconi P, Collins AM, Colin-Jones R, Cutland CL, Darton TC, Dheda K, Duncan CJA, Emary KRW, Ewer KJ, Fairlie L, Faust SN, Feng S, Ferreira DM, Finn A, Goodman AL, Green CM, Green CA, Heath PT, Hill C, Hill H, Hirsch I, Hodgson SHC, Izu A, Jackson S, Jenkin D, Joe CCD, Kerridge S, Koen A, Kwatra G, Lazarus R, Lawrie AM, Lelliott A, Libri V, Lillie PJ, Mallory R, Mendes AVA, Milan EP, Minassian AM, McGregor A, Morrison H, Mujadidi YF, Nana A, O'Reilly
PJ, Padayachee SD, Pittella A, Plested E, Pollock KM, Ramasamy MN, Rhead S, Schwarzbold AV, Singh N, Smith A, Song R, Snape MD, Sprinz E, Sutherland RK, Tarrant R, Thomson EC, Török ME, Toshner M, Turner DPJ, Vekemans J, Villafana TL, Watson MEE, Williams CJ, Douglas AD, Hill AVS, Lambe T, Gilbert SC, Pollard AJ; Oxford COVID Vaccine Trial Group (2020) Safety and efficacy of the ChAdOx1 nCoV-19 vaccine (AZD1222) against SARS-CoV-2: an interim analysis of four randomised controlled trials in Brazil, South Africa, and the UK. Lancet 397:99-111

Wang G, Wu C, Zhang Q, Yu B, LU J, Zhang S, Wu G, Wu Y, Zhong Y (2020) Clinical characteristics and the risk factors for severe events of elderly coronavirus disease 2019 patients. Zhong Nan Da Xue Xue Bao Yi Xue Ban 45:542-548

Weycker D, Edelsberg J, Halloran ME, Longini IM Jr, Nizam A, Ciuryla V, Oster G (2005) Population-wide benefits of routine vaccination of children against influenza. Vaccine 23:1284-1293

Winarski KL, Tang J, Klenow L, Lee J, Coyle EM, Manischewitz J, Turner HL, Takeda K, Ward AB, Golding H, Khurana S (2019) Antibody-dependent enhancement of influenza disease promoted by increase in hemagglutinin stem flexibility and virus fusion kinetics. Proc Natl Acad Sci U S A 116:15194-15199

Yi C, Yi Y, Li J (2020) mRNA Vaccines: possible tools to combat SARS-CoV-2. Virol Sin 35:259-262

Yoda T, Katsuyama H (2021) Willingness to receive COVID-19 vaccination in Japan. Vaccines (basel) 9:48

Zhang C, Maruggi G, Shan H, Li J (2019) Advances in mRNA vaccines for infectious diseases. Front Immunol 10:594 